NOTA TÉCNICA

\title{
CAPTURA DE PARASITOIDES DE Stomoxys calcitrans EN PUPAS DE MOSCA DOMÉSTICA EN COSTA RICA
}

Ligia Rodríguez Rojas ${ }^{1}$

\section{RESUMEN}

Captura de parasitoides de Stomoxys calcitrans en pupas de mosca doméstica en Costa Rica. El objetivo de esta actividad fue recuperar parasitoides de la mosca del establo Stomoxys calcitrans en áreas productoras de piña, utilizando como señuelo pupas de mosca doméstica (Musca domestica) criadas en laboratorio y colocadas en bandejas plásticas sobre el rastrojo de piña. El trabajo se realizó, en plantaciones de piña de dos localidades ubicadas en Pital y Upala, Alajuela, Costa Rica. En Pital, las dos colocaciones de pupas se realizaron del 15 al 27 de setiembre del 2013, con una precipitación total de 209 mm durante el periodo, con temperatura promedio de $26,5^{\circ} \mathrm{C}$ y humedad relativa de $91 \%$. En Upala, las pupas se colocaron del 24 de setiembre al 23 de octubre del 2014. En este caso la precipitación fue de 237,3 mm, temperatura promedio de $25,8^{\circ} \mathrm{C}$ y la humedad relativa de $75,3 \%$. Los parasitoides recuperados en Pital en el primer muestreo fueron: Muscidifurax raptoroides, Spalangia gemina y Aleocharas spp., con tasas de parasitismo aparente de $6,58 \%, 0,17 \%$ y 0,34 \% respectivamente. En el segundo muestreo se recuperaron estos mismos parasitoides con tasas de parasitismo de 1,74 \%, 1,16 \% y 0,15 \%, además se recuperó Eurytoma sivinskii con un parasitismo de 0,32 \%. En Upala, en las fincas Las Brisas y Valle del Tarso se recuperaron Spalangia sp. y Pachycrepoideus sp. con tasas de parasitismo aparente de 0,20 \% y 0,04\% respectivamente. Los parasitoides de mayor interés a ser valorados en el manejo de la mosca del establo, en rastrojo de piña son Spalangia gemina, Muscidifurax raptoroides y Pachycrepoideus sp.

Palabras clave: Muscidifurax raptoroides, Spalangia gemina, Eurytoma sivinskii, Pachycrepoideus sp. y Aleocharas spp.

1 Instituto Nacional de Innovación y Transferencia en Tecnología Agropecuaria, INTA. Costa Rica. Irodriguez@inta.go.cr. Sede del Laboratorio de Servicios de Fitoprotección del INTA. Sabana Sur, San José. 


\section{INTRODUCCIÓN}

La mosca del establo (Stomoxys Calcitrans), es un parásito externo, hematófago, ampliamente distribuido a nivel mundial, que ocupa diversos ecosistemas y es considerado como una plaga de animales domésticos y silvestres (Quiroz 2005, Coronado et al. 2006, Foil y Hogsette 1994). Este insecto causa diferentes daños en el ganado como reducción en la producción de leche hasta $139 \mathrm{~kg}$ anuales, pérdida de peso de 6 a $9 \mathrm{~kg}$ en terneros antes del destete (Taylor et al. 2012), disminución del índice reproductivo y transmisión de enfermedades, también causa anemia a los animales, por la succión continua de sangre (Campbell et al. 2001, Rojas et al. 2003 y Salazar 2008). Además del rastrojo de piña, esta mosca se reproduce en desechos de otros cultivos que proporcionan gran cantidad de materia orgánica en descomposición, como la palma aceitera (Elaeis guineensis) y el banano (Musa paradisiaca) (SFE 2006, SFE 2007).

En Costa Rica, la presencia de esta mosca, en las áreas agro-pastoriles ha afectado principalmente el sector ganadero, de las regiones Huetar Norte y Caribe, al reproducirse en altas cantidades en los desechos de la piña (Ananas comosus) (Rojas et al. 2003, Salazar 2008). El cultivo de piña es uno de los nuevos productos agrícolas con los que Costa Rica ha diversificado sus exportaciones en los últimos años y se siembra en casi todo el territorio nacional e involucra grandes capitales debido a las extensas áreas de cultivo (Acuña 2006). Se estima que actualmente existen alrededor de 40000 hectáreas sembradas de este cultivo, con una productividad bruta de hasta 90 toneladas por hectárea, la cual se exporta principalmente a Europa y Estados Unidos (Aravena 2005, SEPSA 2016)².

Las características biológicas del insecto, como ciclo de vida corto, la alta capacidad reproductiva en los residuos de piña y su alta dispersión, así como

2 SEPSA (Secretaría Ejecutiva de Planificación Sectorial Agropecuaria, CR). 2016. Estudios Económicos e Información. Ministerio de Agricultura y Ganadería, Informe preliminar. la rápida adquisición de resistencia por el uso indiscriminado de agroquímicos, hacen este insecto muy importante desde el punto de vista de salud pública y veterinaria (Vergara y Jiménez 1995). Su difícil control, mediante las estrategias comúnmente utilizadas, hace necesario la búsqueda de otras alternativas, siendo el control biológico mediante parasitoides, una alternativa adicional para el manejo integrado de esta plaga (Coronado et al. 2006, Machtinger et al. 2012).

Existen varias especies de avispas parasitoides que consumen larvas y pupas de esta mosca, reduciendo las poblacionales (Geden et al. 1992, Torres et al. 1994, Crespo et al. 1998, Geden 1999). Se han encontrado los géneros Muscidifurax, Sphalangia, Pachycrepoideus y Eurytoma parasitando pupas de mosca del establo (Costa et al. 2004, Mena et al. 2010).

En el Departamento de Agricultura de los Estados Unidos (USDA), al exponer pupas de mosca común y del establo a los parasitoides Muscidifurax zaraptor, Muscidifurax raptor, Muscidifurax raptorellus y Trichomalopsis sarcophagae, encontraron que la mortalidad de pupas de moscas fue mayor cuando utilizaron Muscidifurax zaraptor, seguido por M. raptor, M. raptorellus y $T$. sarcophagae. El parasitoide que mayor cantidad de pupas de la mosca del establo consumió fue $M$. raptor. Las especies de Muscidifurax encontradas resultaron ser más eficientes para la crianza y la liberación (Lysyk 2004).

En el Sur de California se estudió la abundancia relativa de parasitoides relacionados con la mosca del establo y mosca doméstica, utilizando pupas colectadas en lecherías. Se encontraron siete especies de parasitoides con abundancia de un $90 \%$, con presencia de los parasitoides: Spalangia endius, Spalangia cameroni, Spalangia nigroaenea, Muscidifurax raptor, Muscidifurax zaraptor, Urolepis rufiles y Aleocharas spp., siendo $S$. endius la especie que se presentó en mayor abundancia (19,9 \%) (Petter 1993).

El presente trabajo pretende recuperar parasitoides de la mosca del establo en áreas productoras de piña, utilizando como señuelo pupas de mosca doméstica, criadas en laboratorio. 


\section{MATERIALES Y MÉTODOS}

El trabajo se realizó en plantaciones de piña del distrito de Pital y el cantón de Upala. Estas localidades se encuentran separadas por aproximadamente $100 \mathrm{~km}$ de distancia y pertenecen a la región Huetar Norte. Se ubican a altitudes en un rango de 100 a 200 msnm en zona de vida de Bosque Tropical muy Húmedo. En cada una de las localidades se efectuaron dos colocaciones de pupas señuelo de mosca doméstica. En Pital, las pupas de moscas doméstica se colocaron en la finca La Lydia, del 15 al 27 de setiembre del 2013, mientras que en Upala, se colocaron en las fincas Las Brisas y Valle del Tarso, del 24 setiembre al 23 de octubre del 2014. Los datos climatológicos registrados durante los periodos de colocación de pupas, se pueden observar en el Cuadro 1.

Cuadro 1. Condiciones climáticas presentes en los periodos de colocación de pupas señuelo en las localidades de Pital y Upala. 2013-2014.

\begin{tabular}{l|c|c|c|c|c|c|}
\hline \multicolumn{1}{c|}{ Sitio } & $\begin{array}{c}\text { Precipitación } \\
(\mathbf{m m})\end{array}$ & $\begin{array}{c}\text { Temperatura } \\
\text { mínima }\left({ }^{\circ} \mathbf{C}\right)\end{array}$ & $\begin{array}{c}\text { Temperatura } \\
\text { máxima }\left({ }^{\circ} \mathbf{C}\right)\end{array}$ & $\begin{array}{c}\text { Humedad } \\
\text { relativa (\%) }\end{array}$ & $\begin{array}{c}\text { Radiación } \\
\text { solar mínima } \\
\left(\mathbf{M J} / \mathbf{m}^{2}\right)\end{array}$ & $\begin{array}{c}\text { Radiación } \\
\text { solar máxima } \\
\left(\mathbf{M J} / \mathbf{m}^{2}\right)\end{array}$ \\
\hline Pital, La Lydia & 105,80 & 21,00 & 32,10 & 90,40 & 1,33 & 20,72 \\
\hline Pital, La Lydia & 103,20 & 21,50 & 31,60 & 91,70 & 1,33 & 19,12 \\
\hline Upala, Las Brisas & 188,60 & 16,69 & 31,99 & 72,76 & - & - \\
\hline Upala, Valle del Tarso & 48,70 & 21,63 & 32,88 & 77,85 & - \\
\hline
\end{tabular}

Fuente: Estación meteorológica PINDECO, Pital, San Carlos.

Estación meteorológica Las Brisa, Upala, San Carlos.

\section{Fase de campo}

\section{Recuperación de parasitoides}

Los parasitoides se recuperaron utilizando bandejas plásticas rectangulares de $12 \times 10 \times 10,5 \mathrm{~cm}$, en las que se colocaron pupas de mosca doméstica de uno a dos días de formadas, procedentes del sistema de crianza de INPROTSA y de El Oro. Para obtener pupas de la edad indicada, se retiraron del sistema de crianza larvas del último estadio y se dejaron en reposo en una bandeja hasta el segundo día, para obtener las pupas requeridas. Para conocer la totalidad de pupas llevadas a campo, se procedió a determinar la cantidad por mililitro, las cuales fueron aproximadamente 30 .

En las dos colocaciones realizadas en finca La Lydia, Pital, se utilizaron de 192 a 600 pupas/ bandeja. En las fincas Las Brisas y Valle del Tarso, en Upala, se colocaron de 900 a 1500 pupa/ bandeja respectivamente, dado que es aconsejable utilizar suficiente cantidad de pupas, para asegurar la estimulación de la atracción de los parasitoides (Mexzón 2012)3.

Los lotes utilizados en esta investigación contaban con vegetación circundante, al menos en dos de sus costados, de 1 a $3 \mathrm{~m}$ altura, compuesta por malezas de hoja ancha en floración y algunas Ciperáceas. Esta condición favoreció la presencia de parasitoides en el área de estudio. Las bandejas con las pupas fueron distribuidas en 10 puntos en el área seleccionada, en el borde del lote con rastrojo de piña, previamente triturado (10 días). Estas se colocaron a una altura de $20-30 \mathrm{~cm}$ del suelo, sobre montículos de rastrojo de piña, previamente dispuestos en cada sitio. Los montículos de rastrojo se utilizaron para

3 Mexzón, R. 2012. Emergencia de parasitoides en pupas de mosca doméstica (entrevista). UCR. Comunicación personal. 
concentrar la presencia de la mosca del establo en estos sitios, como un estímulo adicional para los posibles parasitoides presentes en el área (Hall y Fischer 1988).

La colocación de pupas se realizó en las primeras horas de la mañana y se dejaron expuestas durante siete días, para asegurar la parasitación. Para evitar que la lluvia afectara las pupas, se cubrieron las bandejas con una cobertura de plástico negro (techos), colocada a unos 60-70 cm del suelo, con árboles cercanos que les proporcionaban sombra. Las pupas fueron recolectadas y colocadas en bolsas de papel kraft, debidamente rotuladas y luego se llevaron al laboratorio.

\section{Fase de laboratorio}

\section{Emergencia de parasitoides}

En el laboratorio, las pupas se pusieron en frascos de vidrio con tapa a prueba de fuga, cubiertas con tela tergal para permitir la aireación de las pupas y evitar la salida de los parasitoides. Los frascos se mantuvieron durante 40 días a una temperatura de $25^{\circ} \mathrm{C}$ y a humedad relativa de $60-70 \%$ aproximadamente. Antes de abrir los frascos, se colocaron en el congelador por unos minutos para inmovilizar los parasitoides y evitar el escape de los mismos. Luego éstos se colocaron en frascos viales y se rotularon para su respectiva identificación.

Una vez identificados se cuantificaron los parasitoides por género y especies, se calculó el porcentaje de emergencia de los mismos.

\section{RESULTADOS Y DISCUSIÓN}

\section{Géneros de parasitoides recupera- dos en Pital}

En un total de 2961 pupas señuelo expuestas en la primera colocación, las cuales fueron suplidas por el laboratorio de INPROTSA, emergió un total de 220 puparios, de los cuales se recuperaron 210 parasitoides de los géneros: Muscidifurax raptoroides, Spalangia gemina y Aleocharas spp. (Hanson 2013) ${ }^{4}$. La tasa de parasitismo aparente total fue de 7,09\%, con una tasa de emergencia proporcional para los parasitoides encontrados de $6,58 \%$ para Muscidifurax raptoroides, 0,17\% para Spalangia gemina y 0,34\% para Aleocharas spp. Del total de parasitoides emergidos hubo una pérdida de 10 especímenes $(0,34 \%)$ por escape de los frascos de cría. (Cuadro 2). Resultados similares se obtuvieron en un estudio realizado en granjas de varios sitios, en Brasil, donde se recolectaron pupas de mosca doméstica, mosca del establo y Physiphora aenea. En este caso, en 10504 pupas de mosca doméstica colectadas se obtuvo un $7,2 \%$ de parasitoides y un $17 \%$ de larvas de Aleochara puberula. 
Cuadro 2. Emergencia de parasitoides obtenidos, en pupas de (M. domestica) utilizada como señuelo del 15 al 22 de setiembre, Pital, Alajuela. 2013.

\begin{tabular}{|c|c|c|c|c|c|c|}
\hline \multirow[b]{2}{*}{ Muestra } & \multirow{2}{*}{$\begin{array}{l}\text { Pupas mosca } \\
\text { doméstica } \\
\text { colocadas }\end{array}$} & \multirow{2}{*}{$\begin{array}{c}\text { Pupas } \\
\text { parasitadas }\end{array}$} & \multirow{2}{*}{$\begin{array}{l}\text { Parasitoides } \\
\text { obtenidos }\end{array}$} & \multicolumn{3}{|c|}{ Parasitoides recuperados } \\
\hline & & & & $\begin{array}{c}\text { Muscidifurax } \\
\text { raptoroides }\end{array}$ & $\begin{array}{l}\text { Spalangia } \\
\text { gemina }\end{array}$ & Aleocharas spp \\
\hline 1 & 264 & 19 & 17 & 16 & 1 & 0 \\
\hline 2 & 370 & 18 & 16 & 14 & 0 & 2 \\
\hline 3 & 400 & 32 & 32 & 28 & 3 & 1 \\
\hline 4 & 288 & 25 & 25 & 24 & 1 & 0 \\
\hline 5 & 352 & 26 & 26 & 23 & 0 & 3 \\
\hline 6 & 397 & 22 & 21 & 20 & 0 & 1 \\
\hline 7 & 232 & 19 & 19 & 18 & 0 & 1 \\
\hline 8 & 236 & 15 & 14 & 13 & 0 & 1 \\
\hline 9 & 192 & 22 & 19 & 18 & 0 & 1 \\
\hline 10 & 230 & 22 & 21 & 21 & 0 & 0 \\
\hline Totales & 2961 & 220 & 210 & 195 & 5 & 10 \\
\hline \multicolumn{3}{|c|}{ Tasa de parasitismo, \% } & 7,09 & 6,58 & 0,17 & 0,34 \\
\hline
\end{tabular}

La segunda colocación, de 5275 pupas de mosca doméstica, procedente de este mismo laboratorio en esta misma localidad; una semana después de la primera, únicamente $203(3,84 \%)$ de las pupas fueron objeto de parasitación, aproximadamente la mitad del parasitismo encontrado en la primera fecha de colocación. Del total de parasitoides emergidos, un 1,74 \% correspondió a Muscidifurax raptoroides, el 1,16 \% a Spalangia gemina, $0,32 \%$ a Eurytoma sivinskii y 0,15\% a Aleocharas spp. (Cuadro 3) (Hanson 2013)5. Del total de los parasitados emergidos hubo una pérdida de 25 parasitoides $(0,47 \%)$, por escape en los frascos de cría. El parasitoide Spalangia gemina ha sido reportado en la literatura como un parasitoide de las regiones tropicales, con potencial como agente de control biológico contra las moscas domésticas y las que afectan el ganado (Geden 1997, Costa et al. 2004). Este género fue diferenciado genéticamente en Norte América (Taylor et al. 2006) y posteriormente se identificó S. gemina en Pital, Costa Rica (Taylor 2013) ${ }^{6}$.
Estos resultados no difieren de los obtenidos en un estudio similar realizados por Costa et al. (2004), donde se obtuvo una tasa de parasitismo de $(1,6 \%)$ para Spalangia gemina, $(0,3 \%)$ para Phachycrepoideus vindemmiae y de $(0,1 \%)$, para Eurytoma sp.

En cuanto a parasitismo aparente de parasitoides recuperados sobre pupas de moscas; en Venezuela, en 2927 pupas de mosca del establo colectadas en un año, se obtuvo un total de 359 parasitoides de las especies Spalangia cameroni, Muscidifurax raptor, con parasitismo que variaron entre $3,47 \%$ y 43,47 \%; siendo S. cameroni responsable por más del $80 \%$ de los Pteromalidae recuperados en este estudio (Coronado et al. 2006). Mientras que Almeida et al. (1999); en 10500 pupas de mosca doméstica recolectadas en campo obtuvieron un porcentaje de emergencia 1,5\% para Spalangia gemina, 0,11\% para Muscidifurax raptoroides y $0,3 \%$ para Muscidifuras sp.

5 Hanson, P. 2013. Identificación de géneros y especies de parasitoides encontradas en pupas señuelo de mosca doméstica. Museo de Insectos, Universidad de Costa Rica. Comunicación personal.

6 Taylor, D. 2013. Identificación de S. gemina (entrevista). Universidad de Nebraska. Comunicación personal. 
Cuadro 3. Emergencia de parasitoides obtenidos, en pupas de (M. domestica), utilizada como señuelo, del 22 al 27 de setiembre, Pital, Alajuela. 2013.

\begin{tabular}{|c|c|c|c|c|c|c|c|}
\hline \multirow{2}{*}{ Muestra } & \multirow{2}{*}{$\begin{array}{l}\text { Pupas mosca } \\
\text { doméstica }\end{array}$} & \multirow{2}{*}{$\begin{array}{c}\text { Pupas } \\
\text { parasitadas }\end{array}$} & \multirow{2}{*}{$\begin{array}{l}\text { Parasitoides } \\
\text { obtenidos }\end{array}$} & \multicolumn{4}{|c|}{ Parasitoides recuperados } \\
\hline & & & & $\begin{array}{c}\text { Muscidifurax } \\
\text { raptoroides }\end{array}$ & $\begin{array}{l}\text { Spalangia } \\
\text { gemina }\end{array}$ & $\begin{array}{l}\text { Eurytoma } \\
\text { sivinskii }\end{array}$ & $\begin{array}{l}\text { Aleocharas } \\
\text { spp. }\end{array}$ \\
\hline 1 & 514 & 26 & 26 & 5 & 14 & 4 & 3 \\
\hline 2 & 496 & 21 & 21 & 13 & 1 & 7 & 0 \\
\hline 3 & 613 & 49 & 49 & 43 & 6 & 0 & 0 \\
\hline 4 & 604 & 20 & 15 & 3 & 12 & 0 & 0 \\
\hline 5 & 531 & 29 & 22 & 15 & 3 & 3 & 1 \\
\hline 6 & 585 & 10 & 9 & 3 & 4 & 1 & 1 \\
\hline 7 & 487 & 16 & 11 & 9 & 0 & 1 & 1 \\
\hline 8 & 485 & 7 & 3 & 0 & 3 & 0 & 0 \\
\hline 9 & 475 & 14 & 13 & 0 & 12 & 0 & 1 \\
\hline 10 & 485 & 11 & 9 & 1 & 6 & 1 & 1 \\
\hline Totales & 5275 & 203 & 178 & 92 & 61 & 17 & 8 \\
\hline \multicolumn{3}{|c|}{ Tasa de parasitismo, \% } & 3,37 & 1,74 & 1,16 & 0,32 & 0,15 \\
\hline
\end{tabular}

\section{Géneros de parasitoides recupera- dos en Upala}

En la localidad de Upala, en la finca Las Brisas se colocaron 6169 pupas señuelo procedente de la crianza de la empresa Del Oro, se obtuvo un parasitismo total de $0,11 \%$ correspondiente al parasitoide Spalangia sp. Mientras que en finca Valle del Tarso en 9180 pupas señuelo de mosca doméstica se logró recuperar dos géneros, Spalangia sp. y Pachycrepoideus sp. con una tasa total de parasistismo de 0,24 \%. (Cuadro 4). La tasa de parasitismo para Spalangia fue de 0,20 \%, mientras que para Pachycrepoideus sp. fue de $0,04 \%$. Si bien es cierto hay presencia de Spalangia en ambas fincas de esta localidad, su presencia fue baja si la comparamos con los resultados de la localidad de Pital, donde este parasitoide alcanzó una tasa de parasitismo aparente de 1,18 \%. El parasitoide Pachycrepoideus sp. fue recuperado únicamente en la finca Valle del Tarso, en Upala (Cuadro 4). Además de los parasitoides Spalangia cameroni, Spalangia endius y Spalangia gemina, se ha encontrado a Pachycrepoideus vindemmiae y Eurytoma sp. en pupas de la mosca del establo (Costa et al. 2004).

Cuadro 4. Tasa de parasitismo aparente para parasitoide recuperado en pupas de $M$. domestica, del 24 septiembre al 23 de octubre, Upala, Alajuela. 2014.

\begin{tabular}{l|c|c|c|c|c}
\multicolumn{1}{|c|}{ Localidades/parasitismo } & $\begin{array}{c}\text { Total de pupas } \\
\text { de mosca } \\
\text { doméstica } \\
\text { colocadas }\end{array}$ & $\begin{array}{c}\text { Cantidad de } \\
\text { mosca domés- } \\
\text { tica emergida }\end{array}$ & $\begin{array}{c}\text { Total de } \\
\text { parasitoides } \\
\text { emergidos }\end{array}$ & $\begin{array}{c}\text { Spalangia sp. } \\
\text { Pachycrepoideus sp. }\end{array}$ \\
\hline Las Brisas & 6169 & 6162 & 7 & 7 & 0 \\
Tasa de parasitismo, \% & 9180 & 9158 & 0,11 & 0,11 & 0 \\
Valle del Tarso & & & 0,24 & 0,20 & 4 \\
Tasa de parasitismo, \% & & & & 0,04 \\
\hline
\end{tabular}


Bajo las condiciones de producción del cultivo de piña y utilizando pupas de mosca dómestica como señuelo, se recuperaron los parasitoides Muscidifurax raptoroides, Spalangia sp., Spalangia gemina, Pachycrepoideus sp., Eurytoma sivinskii y Aleocharas spp. Los géneros de parasitoides Muscidifurax, Spalangia y Pachycrepoideus han sido reportados a nivel mundial con un alto potencial en el control de dípteros como mosca doméstica y del establo (Jerome et al. 2001), sin embargo en el presente estudio el parasitismo de Pachycrepoideus fue bajo. Es importante resaltar la presencia del género Spalangia recuperado en ambas localidades del estudio, pues además de ser reconocido como parasitoide de la mosca del establo (Mora et al. 1997), es una especie nativa para la región (Taylor 2006), con presencia en áreas donde se cultiva piña.

\section{BIBLIOGRAFÍA}

Acuña, G. 2006. Piña en Costa Rica: producción y ambiente. Ambientico no. 158:1-11.

Almeida, MA; Ferreira, P; Pires, A. 1999. Aleocharas spp y parasitoides de pupas atacan dymbovine volar pupas en el sureste de Brasil. Control Biológico. 14(2):77-83.

Aravena, J. 2005. La Expansión piñera en Casta Rica. La realidad de los perdedores de la agroindustria exportadora de piña. Comunidad Ecologista La Ceiba, Amigos de la tierra. 56 p.

Campbell, JB; Skoda, SR; Berkebile, D; Boxler, DJ; Thomas, GD. 2001. Effects of stable flies (Diptera: Muscidae) on weight gains of grazing yearling cattle. J. Econ. Entomol. 94:780-783.

Coronado, A; Suárez, C; Mujica, F; Henríquez, H. 2006. Parasitoides enemigos naturales de la mosca de los establos, Stomoxys calcitrans, en una finca lechera. Veterinaria Trop. 31(1-2):33-41.

Costa, VA; Berti-Filho, E; Silveira Neto, S. 2004. Parasitóides (Hymenoptera: Chalcidoidea) de moscas sinantrópicas (Diptera: Muscidae) em aviários de Echaporã, sp. Arq. Inst. Biol. 71(2):203-209.

Crespo, D; Lecuona, R; Hogsette, J. 1998. Biological Control: An Important Component in Integrated Management of Musca domestica (Diptera: Muscidae). Biol. Control. 13:16-24.

Foil, L; Hogsette, J. 1994. Biology and control of tabanids, stable flies and horn flies. Sci. Tech. Off. Int. Epiz. 13:1125-1158.
Geden, C; Rutz, D; Scott, J; Long, S. 1992. Susceptibility of house flies (Diptera: Muscidae) and five pupal parasitoids (Hymenoptera: Pteromalidae) to abamectin and seven commercial insecticides. J. Econ. Entomol. 85:435-440.

Geden, C. 1997. Development Models for the Filth Fly Parasitoids Spalangia gemina, S. cameroni, and Muscidifurax raptor (Hymenoptera: Pteromalidae) under constant and variable temperatures. Biological Control. 9:185-192.

Geden, C. 1999. Host location by house fly (Díptera: Muscidae) parasitoids in poultry manure at different moisture levels and host densities. Environ. Entomol. 28:755-760.

Hall, RD; Fischer, FJ. 1988. Laboratory studies on the biology of Spalangia nigra (Hym: Pteromalidae). Entomophaga. 33(4) 495-504.

Jerome, J; Farkas, R; Csaba, T. 2001. Hymenopteran Pupal Parasitoids Recovered from House Fly and Stable Fly (Diptera: Muscidae) Pupae Collected on Livestock Facilities in Southern and Eastern Hungary. Environ. Entomol. 30(1):107-111.

Lysyk, TJ. 2004. Host Mortality and Progeny Production by Solitary and Gregarious Parasitoids (Hymenoptera: Pteromalidae) Attacking Musca domestica and Stomoxys calcitrans (Diptera: Muscidae) at Varying Host Densities. Environ. Entomol. 33(2):328-339. 
Machtinger, E; Leppla, N; Geden, C; Hogsette, J. 2012. Improving biological control on equestrian farms. In: memory 7th International IPM Symposium, Memphis, Estados Unidos. 150 p.

Mena, J; Sivinski, J; Anzures, A; Ramírez, R; Gates, M; Aluja, M. 2010. Consideration of Eurytoma sivinskii Gates and Grissell, a eurytomid (Hymenoptera) with unusual foraging behaviors, as a biological control agent of tephritid (Diptera) fruit flies. Biological Control. 53(1):9-17.

Mora, S; Calvache, H; Alvañil, F; Torres, JA; Verdugo, A; Lun, JE. 1997. La mosca de los establos Stomoxys calcitrans (L.) (Diptera: Muscidae), en palma de aceite. Palmas. 18(3):31-42.

Petter, J. 1993. IPM and Biological Control of Plant pests: Field Crops (Quick bibliography series). United States Department of Agriculture. 55 p.

Quiroz, H. 2005. Parasitología y enfermedades parasitarias de animales domésticos. México D.F. Universidad Nacional Autónoma de México. Editorial Limusa S.A. 714 p.

Rojas, T; Calvo, B; Porras, S; Chavarría, A. 2003. Problemática de la mosca del establo, Stomoxys calcitrans, originada por los desechos del cultivo de la piña (Anannas comosus) en la región Huetar Atlántica de Costa Rica. Ministerio de Agricultura y Ganadería. Boletín de parasitología. 4(1):3-4.

Salazar, O. 2008, Plantaciones de piña en Costa Rica contra la sostenibilidad ecológica y social. Ambientico no. 177. Publicado en Revista Global Hoy, Universidad Nacional, Heredia, Costa Rica. $19 \mathrm{p}$.
SFE (Servicio Fitosanitario del Estado, Costa Rica). 2006. Definen procedimientos en el manejo de desechos orgánicos para el control de (Stomoxys calcitrans L.). San José, Costa Rica. 4 p. Actualidad Fitosanitaria. Boletín no. 28.

SFE (Servicio Fitosanitario del Estado, Costa Rica). 2007. Trituración de desechos orgánicos disminuye poblaciones de mosca del establo. San José, Costa Rica. 4 p. Actualidad Fitosanitaria. Boletín no. 32.

Taylor, D; Moon, R; Gibson, G; Szalanski, A. 2006. Genetic and Morphological Comparisons of New and Old World Populations of Spalangia Species (Hymenoptera: Pteromalidae). Ann. Entomol. Soc. Am. 99(5):799-808.

Taylor, D; Moon, R; Mark, D. 2012. Economic Impact of Stable Flies (Diptera: Muscidae) on Dairy and Beef Cattle Production. J. Med. Entomol. 49(1):198-209.

Torres, P; Cicchino, A; Abrahamovich, AH; Nuñez, J; Prieto, O. 1994. Los enemigos naturales de Haematobia irritans (Diptera: Muscidae) en dos áreas ganaderas de la Argentina. Med. Vet. 75:6-16.

Vergara, R; Jiménez, J. 1995. Manejo integrado de moscas comunes (MIMD) en explotaciones pecuarias y salud pública con énfasis en control biológico. In: Gomero L, Lizárraga A. (eds.). Aportes del Control Biológico en la Agricultura Sostenible. Lima, Perú. p. 347-359. 